\title{
The methodological and conceptual aspects of mortality studies in psychiatry
}

\author{
ANNIBALE BIGGERI and DOLORES CATELAN
}

\begin{abstract}
An extensive literature documented a mortality differential for natural causes between psychiatric patients and the general population. Less clear is the pattern for cancer diseases. Methodological problems arise when trying to explain such mortality gap: selection bias and reverse causation; time-dependent confounders that are also intermediate variables; complex relationships within a life course have to be considered. We try to explain such problems in terms of causal graphs. Excess risk for causes of death which are not attributable to higher prevalence of risk factors or treatment side-effects and higher mortality rates for avoidable causes have been also documented. These findings underline the need for research on health promotion and preventive programs targeted to psychiatric patients.

Declaration of Interest: None.
\end{abstract}

Mortality profiles and life-course studies of psychiatric patients populations are far from having lost their epidemiological appeal. We can list the following open questions:

- What is the specific disease profile of each psychiatric condition? Is it suggestive of peculiar patho-physiological processes?

- What are the main co-morbidities or absence of comorbidities? Are they causally related to treatment?

- Do psychiatric patients suffer inequalities in health? What is the implied web of causation?

The research facing those questions must address major methodological problems: selection bias and reverse causation; time-dependent confounders that are also intermediate variables; complex relationships within a life course.

Psychiatric patients have a higher mortality rate than the general population (Harris \& Barraclough, 1998). The excess is greater in hospital-based cohort studies. Meloni et al. (2006) found a threefold mortality risk in a cohort of psychiatric in-patients in Florence. Grigoletti et al.

Address for correspondence: Dr. D. Catelan, Biostatistics Unit, ISPO Cancer Research and Prevention Institute, Viale Morgagni 59, 50134 Florence (Italy).

Fax: +39-055-3269.7950

E-mail: catelan@ds.unifi.it
(2009) documented that psychiatric patients in contact with South Verona Community-based Mental Health Service have an almost twofold higher mortality rate.

The risk depends on psychiatric diagnosis, gender and age. It is higher for young females, drug addicts or alcoholists (Amaddeo et al., 1995; Prior et al., 1996). Among the top ranking causes of death we found accidents and suicide, infectious diseases, blood diseases, osteo-articular diseases, genito-urinary diseases, nervous system diseases, respiratory, digestive and cardiovascular diseases. The excess for malignant neoplasm is less prominent. (Roshanaei-Moghaddam \& Katon, 2009; Saha et al., 2007).

How can we explain such striking differentials? In fact, the definition of a reference population is far from being straightforward. Psychiatric patients population is composed of people who are mostly excluded from the economically active subset of the general population. Therefore it could be argued that assuming the general population as reference would induce an un-healthy population bias which is symmetrical to the well documented "healthy worker effect" in occupational cohort studies. The "healthy worker effect" depends on the tendency of those working to be selected for their mental and physical ability to work - i.e. being in good health status. For example, economically inactive population shows a relative age-standardized mortality of 3.06 (men) and 2.40 (women) in the 1980's taking fixed at 1.0 the economically active (Martikainen \& Valkonen, 1999). In the studies on the mortality differential by social class, the bias due to the exclusion of economically inactive subset from 
the reference population had been discussed. The consequence was an underestimation of the social gap (Blakely $\&$ Fawcett, 2005). Here we may have the opposite phenomenon. When studying the mortality differential of psychiatric patients it had been the case for bias due to the inclusion of economically active subset in the reference population. The consequence would be an overestimation of the mortality gap. Notice that the abovementioned all cause mortality relative risks for psychiatric patients range between 2 and 3 with respect to the general population, while those attributable to the economically inactive were between 1.5 and 2 (table 1, page 900, in Martikainen and Valkonen, 1999).

This potential distortion should disappear for longer follow-up times (Goldblatt et al., 1990): in the South Verona study we observed a standardized mortality ratio (SMR) of 3.4 in the follow-up period of $\leq 1$ year from first contact, 2.6 during the second year, 1.8 up to five years, 1.5 up to ten years and 1.4 after ten years (Grigoletti et al., 2009). Two comments: first, even at longer follow-up times there still exists a mortality gap; second, how exclusion from economically active population exerts its ill potential has to be clarified.

Mortality for cancer has a distinct pattern. The excess risk for all malignant neoplasm is not as large as it might be expected (Grinshpoon et al., 2005; Barak et al., 2005). But, noteworthy, oral cavity, lymphohematopoietic and central nervous system tumours are in large excess and tumours of digestive system (stomach), breast, prostate were reported less frequently in comparison with the general reference population (Batul et al., submitted for publication).

Those patterns are specific of each psychiatric diagnosis: alcoholism and psychiatric admission for drug addiction are the conditions stronger associated with unfavourable mortality risk. The more plausible explanation is that those patients have risk factors for other diseases, like infectious, digestive system diseases or oral cancer. This explanation is parsimonious, we do not need additional hypothesis. The mortality differential of psychiatric patients cohorts would be the consequence of higher prevalence of exposure to known risk factors in comparison to the general population. There is evidence that prevalence of smoking is lower in the general (healthier) population than among psychiatric patients (Poirier et al., 2002). If we would be able to define a reference population with equal prevalence of smokers and other life habits then the observed mortality disadvantage of psychiatric case population would disappear.

We can generalize this by means of causal graphs (Pearl, 2009). A graph is composed of nodes (variables) and arrows (direct causal relationships). An indirect causal relationship between two variables will exist whenever there is a direct path connecting the two through other connected nodes (i.e. with head-to-tail arrows). Two variables are associated if there is an open path between them. A path is open if there is no collider on it, i.e. two arrowheads meets on a given node. If we condition on a variable (by stratification or other statistical procedures like standardization and multiple regression) which is not a collider we will block the path; if the variable is a collider, conditioning on it will open the path.

Figure 1 illustrates the problem discussed insofar. The selection indicator $\mathrm{S}$ is a collider between $\mathrm{P}(\mathrm{P}=1$ denotes being a psychiatric patient) and $\mathrm{G}(\mathrm{G}=1$ being member of the active population). Clearly Y (survival) depends on $\mathrm{G}$ (population characteristics). Then, conditioning on S, we will observe that $\mathrm{P}$ and $\mathrm{Y}$ (survival) are associated because there is an open path from $\mathrm{P}$ to $\mathrm{Y}$ (see Rothman et al., 2008 for a discussion on healthy worker effect, selection bias and confounding).

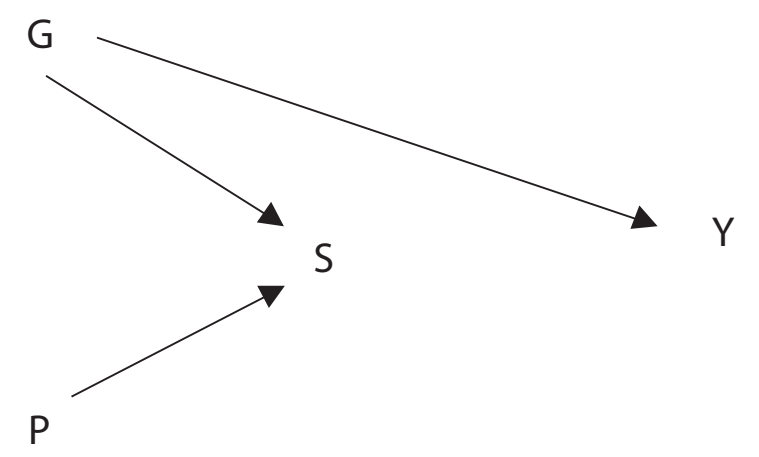

Figure 1 - A causal diagram illustrating a selection bias.

This explanation of the mortality differential is however partial and unsatisfactory. Partial because excess risk for certain diseases is clearly a reverse causation effect: the underlying pathological condition caused the occurrence of psychiatric symptoms (e.g. organic psychosis). Unsatisfactory because the co-morbidity patterns is not entirely consistent with the toxicology of the involved risk factors.

Leaving aside the still unproven idea that schizophrenic patients have a lower cancer risk (Cohen et al., 2002), the relationship between antipsychotic treatment and co-morbidities received recently great attention (Chwastiak, 2009).

Antipsychotic agents of the second generation can induce a metabolic syndrome and therefore constitute a 
risk factor for cardiovascular disorders (Newcomer, 2007). Since they have been widely used for in the last twenty years, psychiatric patients cohorts provided empirical evidence on the risk of mortality for cardiovascular diseases associated to second generation antipsychotic drugs (De Hert et al., 2009). Selection bias may still be an explanation of the observed empirical association, mainly because patients with mental illnesses do have a pre-treatment increased prevalence of risk factors for cardiovascular disease (overweight and obesity, hyperglycemia, dyslipidemia, hypertension, and smoking).

However, adverse metabolic side effects associated with psychotropic medications, such as antipsychotic drugs, were widely documented and several guidelines were diffused (American Diabetes Association et al., 2004). A recent Finnish cohort study (Tiihonen et al., 2009) found a strong heterogeneity of risk among different antipsychotic agents. It is difficult to interpret the comparison with the general population. The study reported a persistent mortality gap. The authors claimed that if a major risk for cardiovascular death be associated to the new generation of antipsychotic drugs it would have been observed an increasing mortality gap. This is a weak argument, indeed, since so many factor and biases may had affected such observation. More interesting, they documented a differential risk among antipsychotic drugs. The statistical analysis used marginal structural models (Robins et al., 2000) addressing potential confounding in the data. Treatment at a given time point depends on previous treatment history, present and past subject conditions. On the other side, we want to assess the causal role of the whole treatment history (cumulative exposure to a given drug) on cardiovascular mortality. The factors which constitute indication for treatment may also be risk factor for cardiovascular disease, and they can therefore confound the association of interest. To proper account for such confounding we need to split the data in a sequence of short time periods and use inverse probability weighting. The weights are proportional to the probability of exposure of interest in each time span. The consequence is that exposed and not exposed periods would be balanced in term of confounding factors restoring a condition usually assured in randomized trials.

In Figure 2 a simple causal graph illustrates the dilemma. Let assume $\mathrm{T}_{\mathrm{k}}$ be the treatment received at time $k, \mathrm{~T}_{\mathrm{k}-1}$ the treatment at time $k-1, \mathrm{Y}$ the outcome of interest, $\mathrm{U}$ an unmeasured confounder and I an intermediate variable. The intermediate variable I is a collider, thus if we condition on it the cumulative effect of $\mathrm{T}$ on $\mathrm{Y}$ will be con-

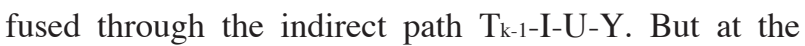

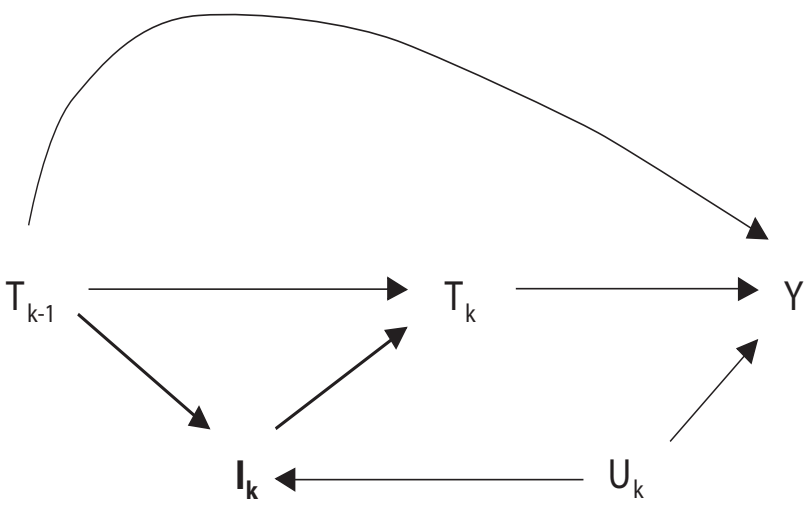

Figure 2 - A causal diagram with a time-dependent factor which is intermediate for previous treatment and confounder.

same time, to estimate the cumulative effect of $\mathrm{T}$ on $\mathrm{Y}$ we must control for I in order to block the indirect path $\mathrm{T}_{\mathrm{k}-\mathrm{I}-}$ U-Y. A solution proposed by Robbins (2000) is to not condition on I and balance $\mathrm{T}_{\mathrm{k}}$ using inverse probability weights proportional to $\operatorname{Prob}\left(\mathrm{T}_{\mathrm{k}} \mathrm{T} \mathrm{T}_{\mathrm{k}-1}, \mathrm{I}\right)$, the probability to receive treatment $T_{k}$ given the past treatment history and the intermediate confounding variable.

Second generation antipsychotic drugs raised also a concern when used in non psychiatric patients, elderly patients and patients with dementia (Wang et al., 2005). Several reports documented a higher risk of all cause mortality. Of course the major menace to the validity of this observation is that the underlying condition which constitutes an indication for treatment is itself a determinant of the disorder leading to death. In the original paper (Wang et al., 2005) instrumental variable technique was used to make causal inference on the risk associated to antipsychotic drugs.

An instrument is a variable which is not associated to the event under study nor it is associated with the confounders but it is a strong predictor of the exposure. A typical instrument is group assignment in a randomized trial. In that study (Wang et al., 2005), the instrument was the physician's prescribing preference and the analysis used the preferred drug as exposure instead of the drug actually prescribed to the patient.

Let concentrate on Figure 3. The instrument variable $\mathrm{Z}$ is strongly associated with treatment $\mathrm{T}$ and it has no effect on the outcome (no direct path pointing to Y). The unmeasured variable $\mathrm{U}$ will confound the association $\mathrm{T}$ $\mathrm{Y}$ (through the open path T-U-Y) but does not confound the association Z-Y (the path Z-T-U-Y is blocked by the collider T). 


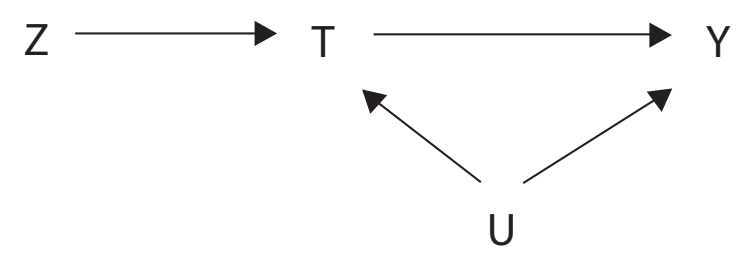

Figure 3 - A causal diagram with an instrumental variable.

In a randomized trial the instrument $\mathrm{Z}$ is treatment assignment. If there is perfect compliance, the association $\mathrm{Z}-\mathrm{Y}$ will be equal to the association T-Y. If not, the compliance indicator $\mathrm{U}$ will confound the T-Y association which can be unbiasedly estimated from the observed not confounded relationships Z-Y and Z-T (Angrist et al., 1996). The same argument applies to observational studies. When an instrument is available we can validly estimate the effect of a treatment in presence of unmeasured confounders, as in the Wang et al. (2005) study.

This illustrates some of the issues in critical appraising the association between treatment received and co-morbidities in a psychiatric cohort study. These warnings are important in order to properly account for reported reduced risk for some cancer sites which were linked to phenothiazines (Lee et al., 2007).

Up to now we commented that psychiatric patients suffer a substantial mortality gap very early in their disease history. There are also documented excesses for some causes of deaths, as reported above, which are not clearly attributable to higher prevalence of risk factors or treatment side-effects. Moreover, higher cardiovascular risk was found in mortality but not using indicators of access to health care. For example, in a Danish study, psychiatric patients had comparable rates of contact for heart disease compared to the general population (Laursen et al., 2009). A possible interpretation is that treatment for heart disease offered to psychiatric patients in Denmark would had been neither sufficiently efficient nor sufficiently intensive. Under-treatment is not only with respect to appropriate management of the disease but more important to management of cardiovascular risk factors (i.e. overweight, diabetes, hypertension).

Some psychiatric patients cohort studies addressed avoidable mortality (Amaddeo et al., 2007). The risk of death for avoidable causes was four times greater than the expected. It should be noticed that the risk was higher for deaths preventable by adequate health promotion policies than for those preventable by appropriate health care.
These findings underline the need for research on health promotion and preventive programs targeted to psychiatric patients.

Recently, strong emphasis in the literature was dedicated to improve capability to manage medical health problems of psychiatric patients by Mental Health Services and Professionals (McIntyre, 2009; Newcomer, 2009).

\section{REFERENCES}

Amaddeo F., Bisoffi G., Bonizzato P., Micciolo R. \& Tansella M. (1995). Mortality among patients with psychiatric illness. A ten-year case register study in an area with a community-based system of care. British Journal of Psychiatry 166, 783-788.

American Diabetes Association, American Psychiatric Association, American Association of Clinical Endocrinologists \& North American Association for the Study of Obesity (2004). Consensus Development Conference on Antipsychotic Drugs and Obesity and Diabetes. Journal of Clinical Psychiatry 65, 267-272.

Angrist J.D., Imbens G.W. \& Rubin D. (1996). Identification of causal effects using instrumental variables. Journal of the American Statistical Association 91, 444-472.

Barak Y., Achiron A., Mandel M., Mirecki I. \& Aizenberg D. (2005). Reduced cancer incidence among patients with schizophrenia. Cancer 104, 2817-2821.

Batul H., Perini G., Grigoletti L., Biggeri A., Tansella M. \& Amaddeo F. (submitted for publication). Cancer mortality among psychiatric patients treated in a community-based system of care: a 25-year case register study.

Blakely T. \& Fawcett J. (2005). Bias measuring mortality gradients by occupational class in New Zealand. New Zealand Medical Journal 118 , U1253.

Chwastiak L.A. (2009). The unchanging mortality gap for people with schizophrenia. Lancet 374, 590-592.

Cohen M., Dembling B. \& Schorling J. (2002). The association between schizophrenia and cancer: a population-based mortality study. Schizophrenia Research 57, 139-146.

De Hert M., Dekker J.M., Wood D., Kahl K.G., Holt R.I.\& Möller H.J. (2009). Cardiovascular disease and diabetes in people with severe mental illness position statement from the European Psychiatric Association (EPA), supported by the European Association for the Study of Diabetes (EASD) and the European Society of Cardiology (ESC). European Psychiatry 24, 412-424.

Goldblatt P., Fox J. \& Leon D. (1990). Mortality of employed men and women. In Longitudinal Study. Mortality and Social Organization (ed. P. Goldblatt), OPCS series LS n. 6. HSMO: London.

Grigoletti L., Perini G., Rossi A., Biggeri A., Barbui C., Tansella M. \& Amaddeo F (2009). Mortality and cause of death among psychiatric patients: a 20-year case-register study in an area with a communitybased system of care. Psychological Medicine 39, 1875-1884.

Grinshpoon A., Barchana M., Ponizovsky A., Lipshitz I., Nahon D., Tal O., Weizman A. \& Levav I. (2005). Cancer in schizophrenia: is the risk higher or lower? Schizophrenia Research 73, 333-341.

Harris E.C. \& Barraclough B. (1998). Excess mortality of mental disorder. British Journal of Psychiatry 173, 11-53.

Laursen T.M., Munk-Olsen T., Agerbo E., Gasse C. \& Mortensen P.B. (2009). Somatic hospital contacts, invasive cardiac procedures, and mortality from heart disease in patients with severe mental disorder. Archives of General Psychiatry 66, 713-720.

Lee M.S., Johansen L., Zhang Y., Wilson A., Keegan M., Avery W., Elliott P., Borisy A.A. \& Keith C.T. (2007). The novel combination of chlorpromazine and pentamidine exerts synergistic antiproliferative effects through dual mitotic action. Cancer Research 67, 11359-11367. 
Martikainen P. \& Valkonen T. (1999). Bias related to the exclusion of the economically inactive in studies on social class differences in mortality. International Journal of Epidemiology 28, 899-904.

McIntyre R.S. (2009). Overview of managing medical comorbidities in patients with severe mental illness. Clinical Psychiatry 70, e17.

Meloni D., Miccinesi G., Bencini A., Conte M., Crocetti E., Zappa M. \& Ferrara M. (2006). Mortality among discharged psychiatric patients in Florence, Italy. Psychiatric Services 57, 1474-1481.

Newcomer J.W. (2007). Metabolic syndrome and mental illness. American Journal of Managed Care 13, S170-S177.

Newcomer J.W. (2009). Comparing the safety and efficacy of atypical antipsychotics in psychiatric patients with comorbid medical illnesses. Journal of Clinical Psychiatry 70, Suppl. 3, 30-36.

Pearl J. (2009). Causality: Models, Reasoning, and Inference $2^{\text {nd }}$ ed. Cambridge University Press: New York.

Poirier M.F., Canceil O., Baylé F., Millet B., Bourdel M.C., Moatti C., Olié J.P. \& Attar-Lévy D. (2002). Prevalence of smoking in psychiatric patients. Progress in Neuropsychopharmacol and Biological Psychiatry 26, 529-537

Prior P., Hassall C. \& Cross K.W. (1996). Causes of death associated with psychiatric illness. Journal of Public Health Medicine 18, 381389.

Robins J.M., Hernan M.A. \& Brumback B. (2000). Marginal structural models and causal inference in epidemiology. Epidemiology 11, 550-560.

Roshanaei-Moghaddam B. \& Katon W. (2009). Premature mortality from general medical illnesses among persons with bipolar disorder: a review. Psychiatric Services 60, 147-156.

Rothman K.J., Greenland S. \& Lash T. (2008). Modern Epidemiology, $3^{\text {rd }}$ ed. Lippincott Williams and Wilkins: Philadelphia, US.

Saha S., Chant D. \& McGrath J. (2007). A systematic review of mortality in schizophrenia: is the differential mortality gap worsening over time? Archives of General Psychiatry 64, 1123-1131.

Tiihonen J., Lönnqvist J., Wahlbeck K., Klaukka T., Niskanen L., Tanskanen A. \& Haukka J. (2009). 11-year follow-up of mortality in patients with schizophrenia: a population-based cohort study (FIN11 study). Lancet 374, 620-627.

Wang P.S., Schneeweiss S., Avorn J., Fischer M.A., Mogun H., Solomon D.H. \& Brookhart M.A. (2005). Risk of death in elderly users of conventional vs. atypical antipsychotic medications. New England Journal of Medicine 353, 2335-2341. 